\title{
Versammlungsfreiheit und Großdemonstrationen
}

Kaum ein Bereich des öffentlichen Rechts ist so wenig geklärt wie das Demonstrationsrecht.' Zwar äußern inzwischen die meisten Kommentierungen Bedenken gegen die Verfassungsmäßigkeit verschiedener Bestimmungen des Versammlungsgesetzes, ${ }^{2}$ doch gehen die Verwaltungs- und Strafgerichte - meistens unausgesprochen ${ }^{3}$ - von der Verfassungsmäßigkeit des Versammlungsgesetzes (VersG) aus. ${ }^{4}$ Eine Rechtsprechung des Bundesverfassungsgerichts zur Versammlungsfreiheit (Artikel 8 I GG) existiert bisher nicht.' Die Klärung wird vielfach zusätzlich erschwert durch eine wirklichkeitsferne Betrachtung insbesondere von Großdemonstrationen, zum Beispiel durch Unkenntnis über das Zustandekommen solcher Demonstrationen, über ihren Ablauf und ihre innere Struktur sowie zum Beispiel über Besonderheiten bei einem Einsatz des Bundesgrenzschutzes. Es wird im folgenden zunächst aufgezeigt, welche Probleme sich insbesondere für Großdemonstrationen aus dem nach meiner Auffassung anachronistischen und verfassungsrechtlich nicht mehr haltbaren Versammlungsbegriff des VersG ergeben; es werden die einschlägigen Bestimmungen des VersG hinsichtlich ihrer Verfassungsmäßigkeit untersucht sowie ferner einige aktuelle Einzelprobleme des Demonstrationsrechts erörtert.

\section{Anachronistischer Versammlungsbegriff des Vers $G$}

Obwohl das VersG - die Grundrechtsschranke der Versammlungsfreiheit nach Artikel 8 II GG - keine Legaldefinition des Versammlungsbegriffes enthält, ${ }^{6}$ postuliert es in mehreren Regelungen verbindlich einen bestimmten Typus von Versammlungen, der gekennzeichnet ist durch eine hierarchische Struktur mit einem bestimmten eindeutig feststehenden Veranstalter und Leiter und verbindlichen Ordnungs- und Weisungsbefugnissen gegenüber Ordnern und Teilnehmern. In $\ 7$ I VersG heißt es: "Jede öffentliche Versammlung muß einen Leiter haben. " Leiter ist der Veranstalter oder die von dem Veranstalter angegebene Person, die für die Leitung der Versammlung »verantwortlich sein soll «?

Auch der »Veranstalter « ist unverzichtbarer Bestandteil des gesetzlichen Versammlungsbegriffs. Wie sich im einzelnen aus $\$ 18$ in Verbindung mit $\$ \$ 7 \mathrm{ff}$. ergibt, ist vorgeschrieben, daß der Leiter den Ablauf der Versammlung bestimmt, daß er während der Versammlung für Ordnung zu sorgen hat, daß er die Versammlung jederzeit unterbrechen oder schließen kann und gegebenenfalls eine unterbrochene Ver-

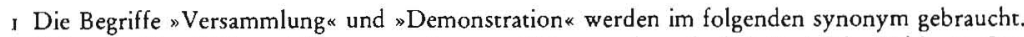

2 Vgl. z. B. Dietel/Gintzel, Demonstrations- und Versammlungsfreiheit $\$ 26$ Rdnr. 8 bis 16 ; Ott, Gesetz über Versammlungen und Aufzüge $\$$ is Rdnr. 2; Vogel, Demonstrationsfreiheit und ihre Grenzen, Schriften der Bundeszentrale für politische Bildung 1969 , S. $23 \mathrm{f}$.

3 Die Ausnahme ist insofern BVerwGE 26, 135 ( $136 \mathrm{ff}$.), das verfassungsrechtliche Ausführungen zur Anmeldepflicht ( I4 $_{4}$ Vers $G$ ) enthält.

4 Vgl. z. B. OLG Köln NStZ 1981, 227; OLG Celle NJW 1977, 444; VGH München NJW 1981, 2428; VGH München NJW 1978, 1939

5 Andeutungen enthält BVerfG NJW 1982, 1803 .

6 Es herrscht im wesentlichen Ubereinstimmung, daß im versammlungsrechtlichen Sinne erforderlich ist eine Versammlung "zu dem Zweck, öffentliche Angelegenheiten gemeinsam zu erörtern oder eine gemeinsame Kundgebung zu veranstalten «; vgl. BVerwGE 26, 135 (137); siehe auch z. B. Dietel/Gintzel, Demonstrations- und Versammlungsfreiheit $\$$ I Rdnr. If

7 Die Bestimmungen des II. Abschnitts des VersG gelten zwar für öffentliche Versammlungen in geschlossenen Räumen, doch sind sie gemäß $\$ 18$ VersG für Versammlungen unter freiem Himmel entsprechend anzuwenden. 
sammlung wieder fortsetzen kann. Er kann ehrenamtliche Order bestellen und ist verpflichtet, falls er sich nicht durchsetzen kann, die Demonstration für beendet zu erklären. Für den einzelnen Teilnehmer gilt, daß er aus der Versammlung ausgeschlossen werden kann und sie dann »sofort zu verlassen « hat; außerdem hat er »die zur Aufrechterhaltung der Ordnung getroffenen Anordnungen des Leiters « zu befolgen.

Diese Festlegung auf einen bestimmten Demonstrationstypus dürfte zusammenhängen mit einer Eigentümlichkeit deutscher Verfassungswirklichkeit, die seit Mitte des vorigen Jahrhunderts durch eine starke Stellung von politischen Organisationen und insbesondere politischen Parteien gekennzeichnet ist, durch die das politische Leben in der Bundesrepublik in einer für westliche Demokratien einzigartigen Weise bestimmt ist. Spätestens seit Mitte des vorigen Jahrhunderts dürften alle wesentlichen größeren Demonstrationen vor allem von politischen Organisationen, Verbänden, Gewerkschaften und Parteien durchgeführt worden sein. Wie groß die Unterschiede zwischen Demonstrationen beispielsweise von Gewerkschaften oder kommunistischen Organisationen einerseits und der politischen Rechten andererseits während der Zeit der Weimarer Republik im einzelnen gewesen sein mögen, waren diese Demonstrationen voneinander doch kaum unterschiedlich hinsichtlich ihrer äußeren Ordnung, der straffen und eindeutigen Demonstrationsleitung und ihrer hierarchischen Struktur.

Auch der Gesetzgeber des VersG von 1953 hatte offenkundig nur diesen Typus straff organisierter Demonstrationen vor Augen. Während das seinerzeit gültige Vereinsgesetz von 1908 beispielsweise "die Wahl des Leiters durch die Versammlung ${ }^{8}{ }^{2}$ zuließ, sollte das neue Gesetz der Demonstrationsführung (Veranstalter, Leiter) vor dem Hintergrund der politischen Verhältnisse der deutschen Nachkriegszeit polizeiähnliche Befugnisse und Verantwortlichkeiten geben. Schon bei der ersten Beratung des damals noch Versammlungsordnungsgesetz genannten späteren VersG im Jahre 1950 erklärte der Bundesminister des Innern zur Begründung des Entwurfes:

»Das zur Zeit noch geltende Vereinsgesetz von 1908 sagt über Versammlungen lediglich, daß der Leiter für die Ordnung verantwortlich ist und daß er eine Versammlung auflösen kann. Das ist nach all den Erfahrungen, die wir seit 1908 und sonderlich in den letzten Jahren gemacht haben, nicht nur zu wenig, sondern falsch akzentuiert; denn wir haben doch grade die Erfahrung gemacht, daß viele Teilnehmer an einer Versammlung es darauf anlegen, sie zu sprengen...

Damit stehen wir bei der besonders wichtigen Figur des Versammlungsleiters. Seine Befugnisse werden gegenüber der unzulänglichen Entfaltung und falschen Akzentuierung im Vereinsgesetz von 1908 in dieser Vorlage näher spezialisiert...

Für den Versammlungsleiter bleibt an der Spitze die Verpflichtung bestehen, für die Ordnung in der Versammlung zu sorgen. An Befugnissen sollen ihm aber nun in näherer Entfaltung gegenüber dem Gesetz von 1908 folgende Befugnisse zugesprochen werden: Er bestimmt den Ablauf der Versammlung. Er kann einen Ordnungsruf erteilen. Er kann das Wort entziehen. Er kann Störenfriede aus der Versammlung ausschließen, und endlich als eine ultima ratio: Er kann die Versammlung auflösen. Die Teilnehmer an der Versammlung sind verpflichtet, sich den Anordnungen des Versammlungsleiters zu fügen und sie zu befolgen. « ${ }^{9}$

Es ist symptomatisch, daß die Vertreter der politischen Linken im damaligen Bundestag - insbesondere die Abgeordneten der SPD und der KPD - den Gesetzentwurf zwar teilweise scharf kritisierten, jedoch hinsichtlich der hierarchisch-disziplinierten und straff organisierten Durchführungsform der Demonstration mit dem Regie-

8 nDer Veranstalter ist berechtigt, die Leitung selbst zu übernehmen, sie einem anderen zu übertragen oder die Wahl des Leiters durch die Versammlung zu veranlassen." $(\$ 10$ Satz 2 des Vereinsgesetzes vom 19. April 1908, GVBl. 1908, 15 1)

9 Beratungen des Deutschen Bundestages, 83. Sirzung I 3123. 
folgendes:

"Wir sind der Meinung, daß die Grundtendenz dieses Gesetzes, Sauberkeit und Ordnung miteinander für das Versammlungsleben zu paaren, zu bejahen ist. Wir sind weiter der Meinung, daß niemandem von diesem Gesetz her Schaden und Gefahren drohen, der sich in eine politisch saubere Praxis einordnet und der Versammlungen und Umzüge in der Form durchführt, wie dies unter anständigen politischen Menschen üblich ist. «10

Insbesondere sprach sich der Vertreter der SPD dafür aus, eine vom Bundesrat vorgeschlagene Stärkung des Versammlungsleiters in den Gesetzentwurf zu übernehmen; geradezu als selbstverständlich ging die SPD davon aus, daß Demonstrationen vornehmlich von Organisationen und politischen Parteien veranstaltet und folglich auch straff organisiert werden können:

"Wenn die Bundesregierung lapidar dazu gesagt hat, hier würden zu hohe Anforderungen an die Versammlungsleiter gestellt, so ist dazu zu sagen, daß das, jedenfalls in dieser allgemeinen Form, nicht ohne weiteres festgestellt werden sollte. Es ist durchaus eine Frage der politischen Erziehung des Funktionärskörpers der politischen Parteien, ob der Funktionär nicht in der Lage ist, den Versammlungsleiter dazu zu bringen, sich so viel Wissen zueigen zu machen, daß er in der Lage ist, zu beurteilen, ob in einer von ihm geleiteten Versammlung die Strafgesetze verletzt werden oder sonstige Vorgänge Grund zum Einschreiten bieten. ""

Es ist demgegenüber offenkundig, daß die größeren Demonstrationen, die spätestens seit Mitte der $70 e r$ Jahre insbesondere gegen die Errichtung umweltschädlicher Großvorhaben stattfinden, von den traditionellen Demonstrationen, deren Bild dem Vers $G$ zugrundeliegt, wesentlich unterschieden sind. Die Initiierung, Vorbereitung und der gesamte Ablauf solcher Demonstrationen wird nicht von Veranstaltern, Leitern und Ordnern bestimmt, sondern vielmehr in häufigen und längerdauernden Diskussionen von vielen Gruppen und Einzelpersonen, die auch in ihrer Gesamtheit keine Veranstalterrolle haben. ${ }^{12}$ Wie groß die Diskrepanz zwischen den Regelungen des VersG und dem faktischen Ablauf solcher Großdemonstrationen ist, wird deutlich, wenn man sich etwa vorstellt, einer der Mitinitiatoren würde während der Demonstration als Leiter auftreten und gegenüber Dritten, die sich nicht an einen vorher verabredeten Demonstrationsablauf halten, gemäß $\$ 10$ VersG Anweisungen geben, sie von der Demonstration ausschließen und sie auf ihre Verpflichtung aus $\$$ I I II VersG hinweisen, daß sie nunmehr die Versammlung "sofort zu verlassen" haben. Hinsichtlich der Verpflichtung des Veranstalters, die beabsichtigte Versammlung »spätestens 48 Stunden vor der Bekanntgabe « $(\$ 14$ VersG) anzumelden, hat das faktische Fehlen von Veranstaltern und Leitern daher dazu geführt, daß diese Anmeldefunktion regelmäßig von kleineren Gruppen - meist einer evangelischen Studentengemeinde oder einem gewerkschaftlichen Arbeitskreis - wahrgenommen wird, denen noch weniger als anderen irgendeine Leitungsfunktion bei der Demonstration zufällt.

10 Beratungen des Deutschen Bundestages, $8_{3}$. Sitzung I 3125

II Beratungen des Deutschen Bundestages, 83. Sitzung I 3 I 25 ; siehe insofern auch die Ausführungen zur zweiten und dritten Lesung des VersG im Jahre 1953, Beratungsniederschriften I $12849 \mathrm{ff}$.

12 Die Polizei hat diesen Wandel übrigens erheblich früher festgestellt als die Rechtswissenschaft und die Gerichte; vgl. z. B. Hübner, Die Polizei in gesellschaftlichem Wandel, in: Die Polizei, 72. Jahrgang, Juni 1982, S. I6s ff.; siehe ferner Berlin-Zürich-Amsterdam, Politik, Protest und die Polizei in: CILIPInformationsdienst, Bürgerrechte und Polizei, Berlin Dezember 1981, S. 2 bis I 57. 


\section{Versammlungsfreibeit als demokratisches Grundrecht}

In Deutschland hat sich das Grundrecht der Versammlungsfreiheit im vergangenen Jahrhundert, verglichen mit anderen westlichen Staaten erst spät und zögernd, herausgebildet. In der Verfassung der Vereinigten Staaten von Nordamerika wurde es bereits im 18 . Jahrhundert durch mehrere amendments (Verfassungsergänzungen) gewährleistet, und zwar gemeinsam mit grundlegenden demokratischen und politischen Rechten wie der Rede- und Pressefreiheit und dem Petitionsrecht. Die Versammlungsfreiheit wurde als naturrechtlicher und vorverfassungsrechtlich geltender Ausdruck der Volkssouveränität und der politischen Partizipation der Bürger am demokratischen Staatsleben überhaupt angesehen. Ähnlich war die Gewährleistung des Versammlungsrechts durch die Gesetzgebungsakte der Französischen Revolution, und zwar als Ausdruck der politischen Meinungsfreiheit bereits in der ersten Deklaration der Bürger- und Menschenrechte vom 26. 8. I 889 sowie ausdrücklich durch Artikel 62 des Gesetzes vom 17. 12. I 889. Zwar ist selbstverständlich, daß die effektive Garantie der Versammlungsfreiheit in diesen Ländern während der letzten 200 Jahre je nach der konkreten politischen und historischen Situation unterschiedlich stark ausgeprägt war, doch war die Versammlungsfreiheit in den angelsächsischen Ländern und in Frankreich von jeweils kurzen Zeiträumen mit stark restaurativen Tendenzen abgesehen bis heute durchgängig als nicht nur individuelles Freiheitsrecht, sondern vielmehr als Bestandteil der Volkssouveränität und der politischen Willensbildung, als »demokratisches" und "politisches» Grundrecht anerkannt. ${ }^{13}$

In Deutschland war demgegenüber die Entwicklung der Versammlungsfreiheit durch zwei entgegengesetzte politische und verfassungsrechtliche Strömungen gekennzeichnet. Während die sogenannte liberale Staatsrechtslehre in Anlehnung an die Traditionen der in Frankreich und den angelsächsischen Ländern entwickelten Grundrechtslehre den politisch-freiheitlichen Charakter der Versammlungsfreiheit betonte, dominierte demgegenüber eine Lehre und Staatspraxis, die im Grunde aus polizeirechtlich-obrigkeitsstaatlicher Sicht die Durchführung von Versammlungen, insbesondere politischen Demonstrationen als Störung der öffentlichen Sicherheit und Ordnung betrachtete und deshalb unter einen generellen Vorbehalt polizeilicher Eingriffe stellte. Die verfassungsrechtlichen Garantien der Versammlungsfreiheit wurden von der Paulskirchen-Verfassung über die Weimarer Reichsverfassung bis zu Artikel 8 des Grundgesetzes ${ }^{14}$ unter Gesetzesvorbehalt gestellt. Welche Bedeutung dieser Gesetzesvorbehalt annahm und wie gering die Substanz der Versammlungsfreiheit demgegenüber war, ist in der Geschichte des deutschen Versammlungsrechts daran zu ersehen, daß in Zeiten politischer Opportunität die Versammlungsfreiheit auf legalem Wege immer wieder fast beliebig eingeschränkt werden konnte. Die wichtigsten Stationen nach der Paulskirchen-Verfassung waren hierbei das PreuBische Versammlungsgesetz von 1850 , das Sozialistengesetz von I 878 , das Vereinsgesetz von 1908, die Notverordnung des Reichspräsidenten von I93 I sowie schließlich die "Verordnung zum Schutze von Volk und Staat« vom 28. 2. 1933.

13 Die ausführlichste Darstellung dieser Zusammenhänge findet sich bei Quilisch, Die demokratische Versammlung, Berlin 1970, S. 30 ff.; siehe auch Schwäble, Das Grundrecht der Versammlungsfreiheit (Art. 8 GG), Berlin 1975 , S. 17 ff. sowie Loening, Vereins- und Versammlungsrecht in: HStW VIII, 1928, S. 542 ff. Siehe ferner Frankenberg, Demonstrationsfreiheit - eine verfassungsrechtliche Skizze, KJ 1981, 370 .

I4 Eine kritische historische Darstellung findet sich bei Blanke/Sterzel, Demonstrationsfreiheit - Geschichte und demokratische Funktion, KJ 198 I, 347 
Obwohl Blanke/Sterzel darin zuzustimmen ist, daß das Versammlungsgesetz von 1953 an diese "staatsautoritären Traditionsbestände « anknüpft, ${ }^{15}$ ist für Artikel 8 I GG immerhin anerkannt, daß das Versammlungsgrundrecht »unverzichtbares Mittel einer aktiven Demokratie ${ }^{16}{ }^{6}$ ist und als »demokratisches Grundrecht « im Zusammenhang mit der Meinungsfreiheit, der Vereinigungsfreiheit und dem Demokratieprinzip (Artikel 5, 9, 20 GG) auszulegen ist und daß der Staat gemäß Art. 8 I GG weitgehende Schutzpflichten für Versammlungen unter freiem Himmel hat und insbesondere verpflichtet ist, diese Versammlungen zu ermöglichen. ${ }^{17} \mathrm{Im}$ wesentlichen knüpfen alle Kommentatoren an die Rechtsprechung des Bundesverfassungsgerichts zu Artikel 20 GG an, derzufolge das Demokratieprinzip einen Prozeß der Meinungs- und Willensbildung des Volkes erfordert, der »sich vom Volk zu den Staatsorganen, nicht umgekehrt von den Staatsorganen zum Volk hin ${ }^{18}$ vollzieht.

Ohne im einzelnen auf die vielfältigen Auslegungsprobleme des Artikels 8 GG einzugehen, ist für die hier erörterten Großdemonstrationen folgendes festzuhalten:

Artikel 8 I GG gewährleistet das Grundrecht, sich »zu versammeln«; der Wortlaut des Grundrechts gibt nichts dafür her, daß solche Versammlungen von Organisationen durchgeführt werden müßten, daß die innere Struktur dieser Versammlung hierarchisch sein müßte und daß ein bestimmter weisungsbefugter und - befähigter Veranstalter oder Leiter einer solchen »Versammlung " verfassungsrechtlich wesensimmanent sei. Auch die Entstehungsgeschichte des Grundrechts ergibt kein anderes Bild. Gegenüber der Formulierung des Herrenchiemseer-Entwurfes wurde zwar aufgrund eines Vorschlags des Grundsatzausschusses ein Gesetzesvorbehalt vorgesehen, doch ergibt sich aus den Protokollen der Entstehungsgeschichte bis zur abschließenden Beschlußfassung nichts, das für eine einschränkende Interpretation des Versammlungsbegriffs sprechen würde, zumal Artikel $8 \mathrm{GG}$ noch während der Geltung des Vereinsgesetzes von 1908 geschaffen wurde, das einen offeneren und liberaleren Versammlungsbegriff vorsieht. Bei den Beratungen des parlamentarischen Rates wurde im wesentlichen über die Frage gestritten, ob Artikel 8 GG ein Deutschen-Recht sein solle sowie über verschiedene redaktionelle Gesichtspunkte. ${ }^{19}$

\section{Verfassungswidrigkeit der Anmeldepflicht}

Die in $\$ 14$ VersG konstituierte Anmeldepflicht ist jedenfalls gegenüber den in diesem Zusammenhang erörterten Großdemonstrationen verfassungswidrig. Nach $₫$ I4 hat derjenige, der die Absicht hat, eine öffentliche Versammlung zu veranstalten, dies spätestens 48 Stunden vor der Bekanntgabe der zuständigen Behörde anzumelden. Die von dem Gesetz unterstellten Voraussetzungen der Anmeldepflicht fehlen in der Regel bei Großdemonstrationen, die pluralistisch initiiert werden und keinen »Veranstalter « kennen, der »die Absicht hat «, eine Demonstration durchzuführen. Es gibt in der Regel auch keine »Person für die Leitung « der Versammlung, welche in der Anmeldung angegeben werden könnte. Auch der Begriff der "Bekanntgabe" kann auf diese Versammlungen nicht zutreffen; diese Versammlungen sind durch die meist öffentlichen und komplexen Diskussionen in der Regel »bekannt « noch bevor Einzelheiten wie Zeitpunkt, Ort und Ablauf der Demonstration bereits feststünden.

Is Blanke/Sterzel, Demonstrationsfreiheit - Geschichte und demokratische Funktion, KJ 1981, 347 (353).

16 So Nawiasky/Leussner, Bayerische Verfassung, Kommentar, S. 195.

17 Vgl. z. B. OVG Lüneburg NJW 1978, 1939; siehe auch Herzog in Maunz/Dürig/Herzog/Scholz, Grundgesetz, Art. 8 Rdnr. 2.

18 BVerfGE 20, 56,2 . Leitsatz; siehe auch BVerfGE 20, $56\left(9^{8} \mathrm{ff}.\right)$.

$19 \mathrm{Vgl}$. JöR n. F. Band I, S. I I 3 ff. 
Die Regelung, daß Demonstrationen vor ihrer Bekanntgabe anzumelden seien, setzt voraus, daß der Veranstalter eine genaue Vorstellung über Zeit und Ablauf der Demonstration hat; in Wirklichkeit ergeben sich die anzumeldenden Tatsachen erst während des Diskussionsprozesses und mithin in der Regel nach ihrem Bekanntwerden.

Darüber hinaus erscheint die Anmeldeverpflichtung auch deshalb als verfassungswidrig, weil Artikel 8 I GG das Recht gewährleistet hat, "sich ohne Anmeldung « zu versammeln. Die in $\$ I_{4}$ Vers $G$ postulierte Anmeldepflicht schränkt diesen Teil des Grundrechts in vollem Umfang ein und ist daher mit Artikel 19 II GG nicht vereinbar. Insofern liegt auch ein wesentlicher Unterschied zu Artikel I23 der WRV vor. In Absatz I dieses Grundrechts war ebenso gewährleistet worden, »sich ohne Anmeldung « zu versammeln, doch umfaßte die Grundrechtsschranke - im Gegensatz zu Art. 8 II GG - auch den Vorbehalt, Versammlungen »anmeldepflichtig « zu machen. Auch die Gesetzesmaterialien sprechen dafür, daß durch den Vorbehalt des Artikels 8 II GG lediglich eine Einschränkung für solche Versammlungen geregelt werden sollte, die nicht "friedlich und ohne Waffen « durchgeführt werden; daß diese Grundrechtsschranke entgegen dem eindeutigen Wortlaut des Artikels 8 I GG auch eine Anmeldung von Versammlungen unter freiem Himmel vorsehen könnte, hat keinem der am Gesetzgebungsverfahren Beteiligten vorgeschwebt. ${ }^{20} \mathrm{Es}$ ist in diesem Zusammenhang darauf hinzuweisen, daß klassische Demokratien ebenfalls grundsätzlich keine Anmeldepflicht kennen. So gelangte Crombach in seiner gründlichen rechtsvergleichenden Untersuchung z. B. zu dem Ergebnis, daß »in der Bundesrepublik Deutschland zwar eine eindeutig erscheinende Regelung existiert, deren Auslegung aber erhebliche Schwierigkeiten bereitet, in England dagegen keine allgemeine Anmeldepflicht normiert ... ist, in der Praxis aber kaum Probleme auftreten. «"1

3. Verfassungswidrigkeit der gesetzlich fixierten bierarchischen Organisationsstruktur

Verfassungswidrig sind jedenfalls hinsichtlich der hier erörterten Großdemonstrationen ferner diejenigen Bestimmungen, die diese Versammlungen auf eine hierarchische Organisationsstruktur festlegen. Dazu gehören insbesondere diejenigen Regelungen, die vorschreiben, daß jede Versammlung einen Leiter haben muß und die diesem Leiter zwingende Ordnungs- und Weisungsbefugnisse und entsprechende

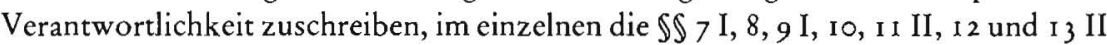
- soweit sie gemäß $\$$ I8 I für öffentliche Versammlungen unter freiem Himmel anzuwenden sind - sowie ferner die $\$ \$$ Ig I-III VersG.

Aus dem gleichen Grunde sind hinsichtlich dieser Demonstrationen die $\$ \$ 25$ und 26 Vers $G$ verfassungswidrig, denenzufolge strafbar ist, wer als Veranstalter oder Leiter eine unzulässige Versammlung durchführt. Dietel/Gintzel ${ }^{22}$ halten im Anschluß an Quilisch ${ }^{23}$ und Denninger ${ }^{24}$ die Bestimmung zumindest teilweise wegen fehlender Tatbestandsmäßigkeit (Artikel I03 II GG) für verfassungswidrig. Verfassungsrechtlich unhaltbar ist die Bestimmung aber bereits deshalb, weil sie der Versammlung Veranstalter und Leiter mit den entsprechenden autoritativen Befugnissen unterstellt

\footnotetext{
$20 \mathrm{Vgl}$. im einzelnen Protokolle der Sitzungen des Hauptausschusses, insbesondere Seiten 616 ff. und $746 \mathrm{ff}$

2 I Crombach, Die öffentliche Versammlung unter freiem Himmel, Berlin 1976, S. $127 \mathrm{ff}$.

22 Dietel/Gintzel, Demonstrations- und Versammlungsfreiheit, $\$ 26$ Rdnr. 8-16.

23 Quilisch, Die demokratische Versammlung, Berlin 1970, S. I 35 ff.

24 Denninger kann ebenso verstanden werden; siehe ders., Zwölf Thesen zur Demonstrationsfreiheit, DRiZ $1969,70 \mathrm{ff}$
} 
und die Strafbarkeit des Veranstalters oder Leiters aus der daraus resultierenden Verantwortung herleitet. Zwar ließe sich argumentieren, wenn eine Großdemonstration keinen Veranstalter und Leiter hat, brauche die Bestimmung daher nicht verfassungswidrig zu sein, sondern es fehle insofern vielmehr bereits am Tatbestand. Dies trifft aber deshalb nicht zu, weil das VersG die Anmeldung der Versammlung durch den Veranstalter ${ }^{25}$ in $\$$ I4 zur Voraussetzung der Rechtmäßigkeit der Versammlung macht; eine rechtmäßige Versammlung ohne Veranstalter ist daher im VersG nicht vorgesehen. Soweit es sich daher nicht um eine zulässige SpontanDemonstration handelt, für die die Rechtsprechung die Anmeldepflicht des $\$$ I4 entgegen dem Wortlaut dieser Bestimmung verneint hat, ist daher ein Veranstalter oder Leiter bei unzulässigen Versammlungen nach dem VersG immer geben und der objektive Tatbestand daher auch insofern begriffsnotwendig erfüllt. Bedenklich ist auch, daß das Gesetz zudem davon ausgeht, daß Veranstalter und Leiter bereits vor Beginn der Demonstration feststehen, obwohl dies bei Großdemonstrationen häufig nicht der Fall ist. ${ }^{26}$

\section{Verfassungswidrigkeit der Verbotsregelung des $\$ Is Vers $G$}

Verfassungsrechtlich problematisch ist ferner die in $\$$ Is geregelte Ermächtigung zum Verbot von Versammlungen im Freien. Der Streit um die Verfassungsmäßigkeit dieser Bestimmung wird bereits seit den 6oer Jahren insbesondere zu der Frage geführt, ob die Verbotsvoraussetzungen in $\$$ Is hinreichend bestimmt sind. ${ }^{27}$ Weitgehende Einigkeit besteht insofern immerhin in einer Kritik der »sehr weit gefaßten unbestimmten Rechtsbegriffe « des VersG und insbesondere seines $\$$ i $9 .{ }^{28}$ Soweit das Problem erörtert wird, herrscht allerdings die Auffassung vor, daß durch die polizeirechtliche Judikatur zum Begriff der »Gefährdung der öffentlichen Sicherheit und Ordnung " eine hinreichende Konkretisierung und Vorhersehbarkeit der Verbotsvoraussetzungen gewährleistet sei. ${ }^{29}$ Diese Auffassung ist jedoch problematisch, weil bei einer polizeirechtlichen Betrachtung dieses Problems die Besonderheiten des

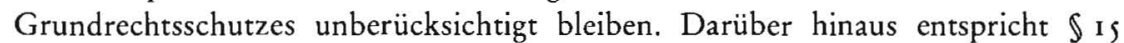
VersG gar nicht der polizeilichen Generalklausel, zu der jene Rechtsprechung ergangen ist, die der Konkretisierung und Auslegung der Verbotsvoraussetzungen dienen soll. Im Gegensatz zu der Generalklausel liegen die Verbotsvoraussetzungen nach $₫$ is erst bei einer »unmittelbaren Gefahr« vor. Wie restriktiv diese Voraussetzung ausgelegt werden sollte, zeigt sich daran, daß der Gesetzgeber ein Vorliegen der Verbotsvoraussetzungen erst annahm, wenn »der Schadenseintritt bei Durchfüh-

25 Eine Ausnahme gilt insofern lediglich für Spontandemonstrationen, die nach herrschender Meinung auch ohne Anmeldung rechtmäßig sind (vgl. z. B. Dietel/Gintzel, Demonstrations- und Versammlungsfreiheit, $\$$ I 4 Rdnr. 18 ff.).

26 Bedenklich daher OLG Köln NStZ I981, 227, wonach der »Leiter « auch verantwortlich sein soll, obwoh er seine Funktion gar nicht mehr wahrgenommen hat. Siehe zu $\$ 26$ VersG auch OLG Düsseldorf NJW 1978, i 8 und BayOLG NJW 1970, 479.

27 Ott, Das Recht auf freie Demonstration, Darmstadt 1967, S. 23 ff.; siehe auch Ott, Gesetz über Versammlungen und Aufzüge, Stuttgart-München-Hannover 1979, $\$$ is Rdnr. I ff.; vgl. hierzu die gründliche Auseinandersetzung bei Hobbeling, Zulässigkeit und Grenzen präventiven Einschreitens gegen Demonstrationen nach $\$$ is Abs. I VersG, Diss. jur. Münster 1973, S. I 8 ff.

28 So Herzog in Maunz/Dürig/Herzog/Scholz, Grundgesetz Art. 8 Rdnr. 98; Herzog kritisiert an gleicher Stelle, daß dadurch »der größte Teil der Verantwortung für die richtige Handhabung des Grundrechts der Polizei in die Schuhe geschoben wird ", hält dies aber »aus praktischen Gründen « für kaum vermeidbar. Unverständlich ist, daß die ambitionierte Kommentierung keine weiteren Ausführungen dazu macht, daß die Verlagerung der Entscheidungen über Demonstrationsverbote auf die Polizei unter dem Gesichtspunkt des Gesetzesvorbehaltes (Art. 8 II in Verbindung mit Art. 20 II und III GG) verfassungsrechtlich problematisch ist.

29 Vgl. z. B. Merten MDR 1968, 624 (625). Siehe auch Dietel, Ermessensschranken bei Eingriffen in das Versammlungs- und Demonstrationsrecht, DVBl 1969, 569 ( $574 \mathrm{f}$.). 
rung der Versammlung (ohne die einschränkenden Verfügungen) mit an Sicherheit grenzender Wahrscheinlichkeit zu erwarten ist $43^{30}$

Für die in diesem Zusammenhang erörterten neueren Großdemonstrationen ergibt sich darüber hinaus ein weiteres Problem. Während $\$$ is die Verbotsvoraussetzungen regelt, fehlt im VersG eine Bestimmung, die die verfassungsrechtlich gebotenen staatlichen Pflichten zum Schutz von Demonstrationen bestimmt, obwohl diese Schutzpflicht von der Rechtsprechung seit einiger Zeit anerkannt wird. Bei friedlich geplanten Großdemonstrationen, auf denen ein nichtfriedliches Auftreten kleinerer Gruppen möglich ist, hat dies in der Praxis dazu geführt, daß diese Demonstrationen zunächst verboten wurden, dann aber dennoch stattfanden und von der Verwaltungsbehörde geduldet wurden. Die Unbestimmtheit der Verbotsvoraussetzungen in $\mathbb{1}$ is sowie das der Behörde bei Verbot und Auflösung eingeräumte Ermessen haben in der Praxis dazu geführt, daß die Verwaltungsbehörden sowohl die Verbotsvoraussetzungen als auch ihr Ermessen über die Auflösung einer verbotenen Demonstration weit auslegen, wodurch die Versammlungsfreiheit zunehmend zur Disposition der Exekutive gestellt wird. Aus diesen Gründen stimme ich der Auffassung Otts zu, daß $\$$ is keine ausreichende gesetzliche Grundlage für Verbot und Auflösung von Versammlungen ist und daher wegen Verletzung der Artikel 8 I und II, 20 II und III GG verfassungswidrig ist.

\section{Der Einsatz des Bundesgrenzschutzes bei Großdemonstrationen}

In den letzten Jahren ist gegenüber Großdemonstrationen zunehmend der Bundesgrenzschutz eingesetzt worden, insbesondere bei Demonstrationen am Standort geplanter atomarer Kraftwerke. Im Raum um Gorleben, wo mehrere Anlagen zur Entsorgung atomarer Kraftwerke errichtet werden sollen, ist der Bundesgrenzschutz seit einiger Zeit zum Schutz dieser Anlagen dauernd stationiert. Es ist bemerkenswert, daß die versammlungsrechtliche Literatur zu diesem Faktum bisher kaum Stellung genommen hat, obwohl diese Einsätze versammlungs- und verfassungsrechtlich problematisch sind und zudem die Rechtmäßigkeit des Einsatzes des Bundesgrenzschutzes in demonstrationsrechtlichen Strafverfahren von Bedeutung ist.

Rechtsgrundlage des Einsatzes des Bundesgrenzschutzes gegenüber Großdemonstrationen ist Artikel 35 II GG in Verb. mit $\$ 9$ I Ziffer I BGSG, wonach ein Land zur Aufrechterhaltung oder Wiederherstellung der öffentlichen Sicherheit und Ordnung »in Fällen von besonderer Bedeutung « Kräfte und Einrichtungen des Bundesgrenzschutzes zur Unterstützung seiner Polizei anfordern kann, »wenn die Polizei ohne diese Unterstützung eine Aufgabe nicht oder nur unter erheblichen Schwierigkeiten erfüllen könnte*. Sowohl Artikel 35 II GG als auch \$9 BGSG waren 1972 unter problematischen Umständen novelliert worden ${ }^{31}$ und erweiterten die $\mathrm{Zu}-$ ständigkeiten des BGS, die von dessen Gründung 1951 bis 1968 lediglich in einem Schutz der Grenzen der Bundesrepublik bestanden hatten und im Zusammenhang mit der Notstandsverfassung I 968 auf den Schutz von Bundesorganen und zur Un-

30 Drucksache des Deutschen Bundestages $8 / 1845$, S. I I.

3I Durch die Novelle wurde eine Tätigkeit legalisiert, die der BGS bereits seit einiger Zeit unter Verletzung der verfassungsrechtlichen Zuständigkeit der Länder praktiziert hatte; vgl. hierzu Geulen, Der Einsatz des BGS zur Sicherung atomarer Anlagen, Strafverteidiger 1981, 306 (308 f.); im Ergebnis ebenso Willich, Historische und aktuelle Probleme der Rechtsstellung des BGS, seine Aufgaben und Befugnisse, Hamburg 1978 (Diss.), S. 227; Maunz in Maunz/Dürig/Herzog/Scholz, Grundgesetz Art. 35, Rdnr. 12; Einwag/Schoen, Bundesgrenzschutzgesetz $\$ 9$ Rdnr. 4 ; siehe auch BT-Drucksache 6/2886, S. 21. 
terstützung der Länderpolizei bei Naturkatastrophen etc. erweitert worden waren.

Da bei der Novellierung der Artikel 35 II GG, $\$ 9$ I Ziff. I BGSG Einigkeit darin bestand, daß dies an der grundsätzlichen Zuständigkeit der Länder zur polizeilichen Gefahrenabwehr (Artikel $30 \mathrm{GG}$ ) nichts ändern sollte, werden die Bestimmungen restriktiv ausgelegt; Voraussetzung eines solchen Einsatzes ist demnach insbesondere, daß die Aufgabe "von besonderer Bedeutung " von dem Land deswegen nicht oder nicht vollständig erfüllt werden kann, weil sie für das Land nicht vorhersehbar ist. ${ }^{32}$ Demnach sind insbesondere dauernde oder stets wiederkehrende Einsätze unzulässig; ein Einsatz, der zunächst rechtmäßig war, kann im Laufe der Zeit unzulässig werden. ${ }^{33}$ Aus den angegebenen Gründen ist der Einsatz des Bundesgrenzschutzes gegenüber Demonstrationen verfassungsrechtlich unzulässig, wenn die von dem Land geltend gemachte Erforderlichkeit zur Erfüllung einer Gefahrenabwehr vorhersehbar war. Ein Dauereinsatz, wie er seit einiger Zeit zum Schutz der geplanten Anlagen im Raum Gorleben praktiziert wird, dürfte jedenfalls inzwischen rechtswidrig geworden sein.

Gegenüber Demonstrationsteilnehmern ergibt sich hieraus zunächst eine Grundrechtsverletzung wegen des im Polizeirecht geltenden Gesetzesvorbehalts, darüber hinaus aber eine Rechtswidrigkeit von Verwaltungsakten, an denen der Bundesgrenzschutz beteiligt ist, und zwar insbesondere einer Auflösung der Versammlung nach $\$ is II VersG, da diese nach dem Wortlaut des Gesetzes nur von der "zuständigen Behörde« vorgenommen werden kann. ${ }^{34}$ Darüber hinaus ergibt sich eine besondere Grundrechtsverletzung aus der Gewährleistung der Versammlungsfreiheit selbst (Artikel 8 I GG). Zwar nimmt der BGS bei diesen Einsätzen polizeiliche Aufgaben für das Land wahr ( $\$$ I 0 III BGSG), doch ist er seit der Novellierung von 1972 eine "Polizei des Bundes « ( $\$ 42$ I BGSG) und unterscheidet sich insbesondere durch die Ausbildung der Dienstleistenden und Angehörigen, ihrer Kasernierung, ihrer Bewaffnung und personellen Zusammensetzung wesentlich von den jeweiligen Landespolizeien. Zudem wurde dem BGS 1972 der sogenannte Kombattantenstatus eingeräumt, und er ist demzufolge mit Beginn eines bewaffneten Konflikts »Teil der bewaffneten Macht der Bundesrepublik Deutschland « ( $\$ 64$ I BGSG); die Vorbereitung auf den Kombattantenstatus ist Teil der Ausbildung des BGS. "s Liegt mithin bei Fehlen der gesetzlichen Voraussetzungen eine Grundrechtsverletzung mangels $\mathrm{Zu}-$ ständigkeit bereits bei einfachen polizeirechtlichen Einsätzen des BGS vor, so besteht erst recht eine Grundrechtsverletzung aus Artikel 8 I GG bei Einsätzen des BGS gegenüber Demonstrationen.

Reiner Geulen

32 Einwag/Schoen, Bundesgrenzschutzgesetz $₫ 9$ Rdnr. 17, BT-Drucksache 6/2886 zu $\$ 9$, S. 25 ; vgl. hierzu auch grundlegend Schnupp, Bundesgrenzschutz: Grenzschutzorgan oder Bundespolizei, DÖD 1972, S. $8 \mathrm{ff}$.

33 Vgl. hierzu neuerdings die gründliche Darstellung von Ernst, Aufgaben und Verwendungsmöglichkeiten des BGS, Göttingen 1980, S. 262 bis 272; ferner Einwag/Schoen, Bundesgrenzschutzgesetz $\$ 9$ Rdnr. 17 .

$34 \mathrm{Daß}$ der BGS insofern gemäß $\$ 10$ III BGSG der Landespolizei unterstellt wird, ändert nichts daran, daß er bei Fehlen der Voraussetzungen des Art. 35 II GG für polizeiliche Eingriffe unzuständig ist.

$35 \mathrm{Vgl}$. hierzu die Auseinandersetzungen um den Kombattantenstatus des BGS bei den Beratungen der Novelle 1972 des BGSG in BT-Protokolle, $3_{8} 3$. Sitzung vom 7. Juli 1972, S. 601 ; siehe auch Geulen, Der Einsatz des Bundesgrenzschutzes zur Sicherung atomarer Anlagen, Strafverteidiger 198 I, 306 (309). 